\title{
Myelination of the right parahippocampal cingulum is associated with physical activity in young healthy adults
}

\author{
Tobias Bracht $^{1,2,3} \cdot$ Derek K. Jones ${ }^{1,2} \cdot$ Sonya Bells $^{1,2} \cdot$ Sebastian Walther $^{3} \cdot$ \\ Mark Drakesmith $^{1,2} \cdot$ David Linden ${ }^{1,2,4}$
}

Received: 30 May 2015/Accepted: 5 January 2016/Published online: 19 January 2016

(c) The Author(s) 2016. This article is published with open access at Springerlink.com

\begin{abstract}
Recent evidence suggests that individual differences in physical activity (PA) may be associated with individual differences in white matter microstructure and with grey matter volume of the hippocampus. Therefore, this study investigated the association between PA and white matter microstructure of pathways connecting to the hippocampus. A total of 33 young, healthy adults underwent magnetic resonance imaging (MRI). High angular resolution diffusion-weighted imaging and multi-component relaxometry MRI scans (multi-component driven equilibrium pulse observation of T1 and T2) were acquired for each participant. Activity levels (AL) of participants were calculated from 72-h actigraphy recordings. Tractography using the damped Richardson Lucy algorithm was used to reconstruct the fornix and bilateral parahippocampal cinguli (PHC). The mean fractional anisotropy (FA) and the myelin water fraction (MWF), a putative marker of myelination, were determined for each pathway. A positive correlation between both AL and FA and between AL and MWF were hypothesized for the three pathways. There was a selective positive correlation between AL and MWF in
\end{abstract}

Tobias Bracht

bracht@puk.unibe.ch

1 Cardiff University Brain Research Imaging Centre (CUBRIC), School of Psychology, Cardiff University, Cardiff, UK

2 Neuroscience and Mental Health Research Institute (NMHRI), Cardiff University, Cardiff, UK

3 Translational Research Centre, University Hospital of Psychiatry, University of Bern, Bolligenstrasse 111, 3000 Bern, Switzerland

4 MRC Centre for Neuropsychiatry Genetics and Genomics, School of Medicine, Cardiff University, Cardiff, UK the right PHC ( $r=0.482, p=0.007)$. Thus, our results provide initial in vivo evidence for an association between myelination of the right PHC and PA in young healthy adults. Our results suggest that MWF may not only be more specific, but also more sensitive than FA to detect white matter microstructural alterations. If PA was to induce structural plasticity of the right PHC this may contribute to reverse structural alterations of the right $\mathrm{PHC}$ in neuropsychiatric disorder with hippocampal pathologies.

Keywords White matter - Diffusion tensor imaging · Actigraphy · Activity · Hippocampus · Neuroplasticity

\section{Introduction}

\section{Physical activity and white matter microstructure}

The benefits of physical activity (PA) for physical and mental health are well established (Hillman et al. 2008; Soundy et al. 2015). Meta-analyses in children suggest that higher PA is associated with improved cognitive performance (Sibley and Etnier 2003; Fedewa and Ahn 2011). For instance, a cross-sectional study found that higher PA is associated with better performance in executive functioning in children (van der Niet et al. 2014). PA has also been consistently reported to induce pleasurable affective responses (Ekkekakis et al. 2005; Bartlett et al. 2011). Cognitive performance and pleasure are also linked to brain structure (Fields 2008; Johansen-Berg 2010; Bracht et al. 2015a). Consequently, there has been an increasing interest in the effect of PA on the structure of the brain.

Diffusion tensor magnetic resonance imaging (DT-MRI) enables a non-invasive in vivo assessment of brain white matter microstructure (Basser et al. 1994). Diffusion 
properties such as fractional anisotropy (FA) indirectly reflect the extent to which the diffusion of water molecules in the brain tissue is preferentially hindered along one direction compared to others, which in turn provides information on the underlying white matter microstructure (Basser and Pierpaoli 1996). A previous DT-MRI study found a positive correlation between aerobic fitness and FA in the uncinate fasciculus and cingulum bundle (Marks et al. 2007). Findings in segments of the middle cingulum were replicated by the same group using objective markers of aerobic fitness (Marks et al. 2011). Walther et al. (2010), using $24 \mathrm{~h}$ recordings of actigraphy as an objective measure of motor activity to assess PA, found a positive association between activity levels (AL) and FA in the right superior longitudinal fasciculus (SLF) and cingulum bundle, and a negative correlation between $\mathrm{AL}$ and FA in the left corticobulbar tract, right posterior corpus callosum and left SLF (Walther et al. 2010). Further, associations between PA and white matter microstructure were identified in the corticospinal tract (Herting et al. 2014), the corpus callosum (Johnson et al. 2012) and in prefrontal, parietal and temporal brain regions (Voss et al. 2013). Thus, there is converging evidence that differences in PA are associated with differences in white matter microstructure in multiple brain regions.

\section{Hippocampal pathways, physical activity and clinical implications}

In addition to brain regions mentioned above, the hippocampus is a further region of particular interest regarding the structural correlates of PA. Animal research suggests strongly that PA induces neuroplastic processes in the hippocampus. For example, it has been shown that wheelrunning in mice increases cell proliferation in the dentate gyrus (van Praag et al. 1999a, b, 2005), and induces increases in brain-derived neurotrophic factor (BDNF), which supports survival of neurons, localized in hippocampal areas (Berchtold et al. 2005; Neeper et al. 1995). BDNF has been also shown to induce myelination in white matter pathways in both animal and in in vitro studies (Wong et al. 2014; Xiao et al. 2010). In elderly humans, PA was associated with increased volumes in bilateral hippocampi (Erickson et al. 2009). This finding was corroborated by findings that a PA exercise program (in contrast to a stretching program) led to increases in volume in the hippocampus (Erickson et al. 2011). Moreover, mean diffusivity (MD) has been shown to be reduced in elderly master athletes in a region incorporated in the parahippocampal cingulum in comparison with a less fit agematched control group (Tseng et al. 2013), while a reduction in FA was reported in sedentary older adults in a recent DTI study (Burzynska et al. 2014).
Structural alterations of the hippocampus may not only be related to PA. Additionally, structural alterations of the hippocampus and its connection pathways have been reported consistently in neuropsychiatric disorders such as depression (Campbell and MacQueen 2004), schizophrenia (Heckers 2001) or dementia (den Heijer et al. 2010). Interestingly, PA may improve symptoms in aforementioned neuropsychiatric disorders (Mead et al. 2009; Heyn et al. 2004; Rosenbaum et al. 2014; Malchow et al. 2015). Thus there may be an association between PA, structural remodelling of hippocampal pathways and clinical recovery. This assumption is supported by a longitudinal study in major depressive disorder (MDD) identifying age-dependent white matter microstructural changes to occur in the left PHC during the time-span from depression to remission in a sample that also significantly increased their $\mathrm{AL}$ (Bracht et al. 2015b). In this study we exclusively focus on the pathways connecting to the hippocampus because these pathways may not only be associated with PA but PA may also impact on structural alterations in neuropsychiatric disorders such as schizophrenia, depression or dementia thereby potentially reversing structural alterations and leading to clinical improvements.

\section{Diffusion properties and myelin water fraction}

Most previous studies using DT-MRI-based diffusion properties such as FA for the assessment of white matter microstructure are limited by the lack of specificity for white matter sub-compartments. For instance, increases in FA may stem from reductions in axonal diameter, higher axonal density, higher myelination, and/or lower intravoxel orientational dispersion (Beaulieu 2002; Jones et al. 2013b). Thus, DT-MRI-based measures on their own do not allow for a sub-compartment specific, and therefore neurobiologically meaningful, interpretation of the data. Moreover, the lack of specificity may lead to conflicting results across studies. For instance, while greater myelination and larger axonal diameter both increase conduction velocity, they have opposite effects on FA.

The development of multicomponent driven equilibrium single pulse observation of $\mathrm{T} 1$ and $\mathrm{T} 2$ (McDESPOT) allows for rapid acquisition of data to produce whole brain myelin water fraction (MWF) maps which have been shown to correlate with myelination (Deoni et al. 2008a; Hurley et al. 2010; Laule et al. 2006; Moore et al. 2000; MacKay et al. 1994). One previous study used MWF as a marker of myelination and found a spatial pattern of myelination in infants that showed striking similarities with what is known from post-mortem studies (Deoni et al. 2011). In addition, studies in demyelinating neurological disorders such as multiple sclerosis strongly suggest the validity of MWF as a measure for myelination (Kolind 
et al. 2012; Kitzler et al. 2012). In one longitudinal study during early childhood changes of MWF correlated positively with performance measures such as gross motor behaviour, visual reception and receptive language (Dean et al. 2014). Finally, the most compelling evidence for the assumption that MWF indeed measures myelin stems from comparisons between MWF maps derived from a shaking pup myelin mutant and control animals (Hurley et al. 2010) and imaging studies demonstrating correlations between MWF and histopathology in multiple sclerosis (Laule et al. 2006; Moore et al. 2000). Consequently, there is ample evidence that MWF does correlate with myelination, suggesting that this measure may represent a useful marker to detect associations of brain structure and function. Therefore, the measure of MWF represents a significant step forward for the interpretability of previously reported changes in white matter, compared to reliance on DT-MRI alone.

\section{Aims of the study and hypothesis}

The present study explored the association between PA and white matter microstructure of the two main hippocampal pathways: the fornix and the PHC. The fornix is the main efferent pathway of the hippocampus projecting to the mammillary bodies (Nieuwenhuys et al. 2007). The PHC forms part of the cingulum bundle and contains predominantly afferent projections from the posterior parietal cortex (Goldman-Rakic et al. 1984; Mufson and Pandya 1984; Jones et al. 2013a). In the present study we use FA as a well-established (though unspecific) marker of white matter microstructure. Further, we use MWF as a potentially more specific measure of myelination.

We hypothesize a positive correlation between FA and $\mathrm{AL}$, and a positive correlation between MWF and AL, in the fornix and in bilateral PHC. We assume that due to the specificity for myelination, i.e., being less susceptible to confounding microstructural differences such as axonal diameter, density and axonal orientational dispersion than DT-MRI metrics, (De Santis et al. 2014) MWF will be a more sensitive marker than FA to detect associations between individual differences between PA and white matter microstructure.

\section{Methods}

\section{Participants}

All participants were recruited through the School of Psychology, Cardiff, Wales, UK. All participants were undergoing or had previously completed a university degree course, were right handed as assessed with the
Edinburgh Handedness Inventory (Oldfield 1971) and of Caucasian origin. Exclusion criteria were a current episode or a history of neurological and psychiatric disorders, drug or alcohol abuse and medication that may have an impact on the structure of the brain. For assessment, the general health questionnaire was used (Goldberg and Huxley 1980). Since training may impact the structure of the brain we also excluded professional athletes, musicians and those at competitive amateur sport levels (Scholz et al. 2009; Bengtsson et al. 2005; Hanggi et al. 2010). A total of thirtythree participants was recruited (19 female, 14 male). Participants had a mean age of $25.5 \pm 4.2$ years. All participants provided written informed consent in order to take part in the study and received monetary compensation. The study had been approved by the local ethics committee of the School of Psychology, Cardiff (EC.13.07.02.3491RA).

\section{Actigraphy}

Participants wore an Actiwatch (Cambridge Neurotechnology, Inc., Cambridge, UK) on the left wrist for 72 consecutive hours. Activity counts were stored in $5 \mathrm{~s}$ intervals. Participants provided an activity protocol stating the kind of daily activities and wake time. Activity levels (AL) (the cumulated activity counts during wake time divided by the net recording time in hours) were calculated separately for each day. Activity analyses were restricted to wake time. Mean-AL was calculated by averaging AL over the three consecutive days. The left wrist was chosen because AL of the non-dominant arm reflects whole body movements without impact of manual fine motor activities (Middelkoop et al. 1997). Protocols were checked for consistency between reported activities and AL measures. Almost identical approaches have been repeatedly used in previous studies e.g. (Bracht et al. 2012; Walther et al. 2009; Razavi et al. 2011).

\section{Structural MRI scanning}

$\mathrm{T}_{1}$-weighted structural scans were acquired using an oblique axial, 3D fast-spoiled gradient recalled sequence (FSPGR) with the following parameters: $\mathrm{TR}=7.9 \mathrm{~ms}$, $\mathrm{TE}=3.0 \mathrm{~ms}$, inversion time $=450 \mathrm{~ms}$, flip angle $=20^{\circ}$, $1 \mathrm{~mm}$ isotropic resolution, with a total acquisition time of approximately $7 \mathrm{~min}$.

\section{Diffusion MRI scanning}

High angular resolution diffusion-weighted imaging (HARDI) data were acquired in the Cardiff University Brain Research Imaging Centre (CUBRIC) on a $3 \mathrm{~T}$ GE Signa HDx system (General Electric, Milwaukee, USA) using a cardiac-gated peripherally gated twice-refocused 
spin-echo Echo Planar Imaging (EPI) sequence, with effective TR/TE of $15 R-R$ intervals $/ 87 \mathrm{~ms}$. Sets of 60 contiguous $2.4 \mathrm{~mm}$ thick axial slices were obtained, with diffusion-sensitizing gradients applied along 30 isotropically distributed (Jones et al. 1999) gradient directions ( $b$ value $\left.=1200 \mathrm{~s} / \mathrm{mm}^{2}\right)$. The field of view was $23 \times 23 \mathrm{~cm}$; and the acquisition matrix was $96 \times 96$, resulting in data acquired with a $2.4 \times 2.4 \times 2.4 \mathrm{~mm}$ isotropic resolution. Following zero-filling to a $128 \times 128$ in-plane matrix for the fast Fourier transform, the final image resolution was $1.8 \times 1.8 \times 2.4 \mathrm{~mm}$. A parallel acceleration (ASSET) factor of 2 was used. Acquisition time was approximately $12 \mathrm{~min}$.

\section{McDESPOT scanning}

The McDESPOT protocol consists of a combination of sagittally oriented spoiled gradient recalled (SPGR), balanced steady-state free procession (bSSFP) and inversionrecovery prepared SPGR (IR-SPGR) sequences (Deoni et al. 2008c, d). All three sequences were acquired with a FOV of $220 \mathrm{~mm} ; 1.7 \mathrm{~mm} \times 1.7 \mathrm{~mm} \times 1.7 \mathrm{~mm}$ voxels, with frequency encoding in the superior-inferior direction for a total scan time of approximately $12 \mathrm{~min}$. mcDESPOT protocol: SPGR, acquisitions: $\mathrm{TE}=2.1 \mathrm{~ms}, \mathrm{TR}=4.7 \mathrm{~ms}$, flip angles $=\left[3^{\circ}, 4^{\circ}, 5^{\circ}, 6^{\circ}, 7^{\circ}, 9^{\circ}, 13^{\circ}, 18^{\circ}\right]$; bSSFP acquisitions: $\quad \mathrm{TE}=1.6 \mathrm{~ms}, \quad \mathrm{TR}=3.2 \mathrm{~ms}, \quad$ flip angles $=\left[10.6^{\circ}, 14.1^{\circ}, 18.5^{\circ}, 23.8^{\circ}, 29.1^{\circ}, 35.3^{\circ}, 45^{\circ}\right.$, $60^{\circ}$ ]. bSSFP acquisitions were repeated with and without $180^{\circ} \mathrm{RF}$ phase alteration to remove SSFP banding artefacts and SPGR and IR-SPGR acquisitions were used to correct $\mathrm{B} 0$ and B1-induced errors in the derived MWF estimates.

\section{McDESPOT data pre-processing}

SPGR and bSSFP images for each participant were linearly coregistered using an affine (12 degrees of freedom) technique based on mutual information to the first image in the sequence to correct for interscan and intrascan motion (Jenkinson and Smith 2001). SPGR and IR-SPGR images were used for DESPOT1 with High-speed Incorporation (DESPOT1-HIFI) of RF Field Inhomogeneities processing as described in (Deoni et al. 2006b; Deoni 2007), resulting in $B_{1}$ field and quantitative $T_{1}$ maps. These $B_{1}$ field and $T_{1}$ maps were used in the subsequent calculation of $B_{0}$ field and $\mathrm{T}_{2}$ maps using two phase-cycled bSSFP data using the DESPOT2 with full modeling (DESPOT2-FM) algorithm (Deoni et al. 2004). Combining SPGR, IR-SPGR and SSFP sequences allowed for the estimation of the multi-component three pool DESPOT model (Deoni et al. 2008b, c, 2013). Alongside other metrics not studied here (myelin water residence time and intra- and extra-cellular (IE) water and myelin water $T_{1}$ and $T_{2}$ ), this model provides whole brain estimates of the myelin water fraction (MWF). $R_{1}$ maps were computed by taking the reciprocal of $T_{1}$.

\section{Post-processing of McDESPOT data}

A synthetic- $T_{1}$ image was computed from the quantitative $\mathrm{T}_{1}$ map (arising from the DESPOT1 data) for each subject assuming the same imaging parameters used to generate the FSPGR T1-weighted image (Deoni et al. 2006a). This effectively creates a template in MWF space with the same contrast as the target $T_{1}$-weighted image. The synthetic $T_{1^{-}}$ weighted image of each participant was then warped to the corresponding $\mathrm{T}_{1}$-weighted structural scan using the FNIRT non-linear registration tool (Jenkinson et al. 2002). The computed warps were then applied to the MWF map and to the quantitative $T_{1}$ map (which is being used to calculate $R_{1}$ ) to transform those maps into the same space as the structural $\mathrm{T}_{1}$-weighted image.

\section{Diffusion MRI data pre-processing}

Data were analysed using ExploreDTI 4.8.3 (Leemans et al. 2009). Eddy-current induced distortion and motion correction was performed using an affine registration to the non-diffusion-weighted $\mathrm{B}_{0}$-images, with appropriate reorienting of the encoding vectors (Leemans and Jones 2009). Field inhomogeneities were corrected for using the approach of (Wu et al. 2008). The diffusion weighted images (DWIs) were non-linearly warped to the $\mathrm{T}_{1^{-}}$ weighted image using the FA map, calculated from the DWIs, as a reference. Warps were computed using Elastix (Klein et al. 2010) using normalized mutual information as the cost function and constraining deformations to the phase-encoding direction. The corrected DWIs were therefore transformed to the same (undistorted) space as the $\mathrm{T}_{1}$-weighted structural images. A single diffusion tensor model was fitted to the diffusion data in order to compute quantitative parameters such as FA (Basser et al. 1994). Following the method of Pasternak et al. (MetzlerBaddeley et al. 2012; Pasternak et al. 2009) a correction for free water contamination of the diffusion tensor based estimates was applied (Metzler-Baddeley et al. 2012; Pasternak et al. 2009). Data quality was checked by careful visual inspection and by looking at the average residuals per DWI for each participant.

\section{Tractography}

Tractography was performed using ExploreDTI (Leemans et al. 2009). Whole brain deterministic tractography was performed following peaks in the fibre orientation density function (fODF) reconstructed from the damped Richardson Lucy algorithm (dRL) (Dell'acqua et al. 2010; 
Jeurissen et al. 2013). The dRL algorithm estimates multiple fibre orientations in a single voxel and therefore provides a more accurate diffusion profile than it is the case for DT-MRI-based methods estimating only one fibre orientation per voxel. For each voxel in the dataset, streamlines were initiated along any peak in the fibre orientation density function (fODF) that exceeded an amplitude of 0.05. A streamline, uniform step-size, algorithm based on that of (Basser et al. 2000), but extended to multiple fibre orientations within each voxel (Jeurissen et al. 2011) was used for tractography. Each streamline continued in $0.5 \mathrm{~mm}$ steps following the peak in the fODF that subtended the smallest angle to the incoming trajectory. Termination criteria were an angle threshold $>45^{\circ}$ and fODF amplitude $<0.05$.

\section{Tract reconstruction}

Tract 'waypoint' regions were drawn manually by one experimenter (T.B.) based on anatomical landmarks. The fornix and bilateral PHC (see Figs. 1,2) were reconstructed according to previously described algorithms (Bracht et al. 2015b; Metzler-Baddeley et al. 2013; Jones et al. 2013a). For reconstruction of the fornix, a coronal region of interest (ROI) was placed around the columns of the fornix four slices posterior to the anterior commissure (Bracht et al. 2015b). For reconstruction of bilateral PHC one horizontal ROI was placed at the height of the most ventral point of the splenium, and a second ROI was placed four slices above. A NOT ROI was placed above the body of the corpus callosum caudal to the rostral-caudal midpoint of the body of the corpus callosum as described in (MetzlerBaddeley et al. 2013; Jones et al. 2013a). For each subject, the anatomical course of each tract was checked carefully. Mean-FA was derived for each reconstructed tract. In addition, the average mean diffusivity (MD) and axial and radial diffusivity (AD, RD) were computed for each tract. Further, mean-MWF and R1 were sampled along the tracts. Those were derived from the MWF-and the single T1image respectively that had been warped to the $T_{1}$ weighted structural image.

\section{Statistical analyses}

Statistical analyses were performed using SPSS22 (SPSS, Inc., Chicago, IL, USA). First, normal distribution of mean-AL, FA and MWF-values was confirmed using Shapiro-Wilk-Tests. Second, Pearson correlations between the mean-AL and FA of bilateral PHC and the fornix were calculated. Third, correlations between mean-AL and MWF, our measure for myelination, were calculated for each of the three hippocampal pathways. We applied a strict Bonferroni correction for multiple comparisons. Thus the level of significance was set at $p<0.0083$ (0.05 divided by the number of tests, $n=6$ ). In pathways where significant correlations were detected we additionally controlled for age and gender calculating separate partial correlation with age and gender as covariates. Further, significant correlations between AL and FA were followed up using MD, RD and AD. Significant correlations between AL and MWF were followed up using R1.

\section{Results}

\section{Activity levels}

One participant had to be excluded from the analyses because their actigraphy recording was incomplete and there had been inconsistencies between reported activities and recorded AL. Thus a total of 32 participants with ALrecordings remained. AL was normally distributed for each of the three single days and for mean-AL (averaged over the 3 days). Repeated measure analyses revealed no significant differences of $\mathrm{AL}$ for the three consecutive days $[F(2,30)=0.37, p=0.70]$. AL-values were as follows: AL day $1=20,513 \pm 6081$, AL day $2=21,496 \pm 8000$, AL day $3=21,516 \pm 6174$, and mean$\mathrm{AL}=21,174 \pm 5345$. Men and women did not differ regarding $\mathrm{AL}$ for any of the days or regarding mean-AL.

\section{Correlations between activity levels and white matter microstructure}

For each of the tracts, FA and MWF values were normally distributed. Across the 32 participants there were no significant correlations between mean-AL and FA for the fornix $\quad(r=0.232, \quad p=0.201), \quad$ left $\quad(r=-0.123$, $p=0.503$ ) or right $(r=0.047, p=0.800)$ PHC.

Two participants had to be excluded from the McDESPOT analyses because of incomplete data acquisition, and thus 30 scans remained. There was a positive correlation between mean-AL and MWF for the right $(r=0.482$, $p=0.007$ ) but not for the left ( $r=0.069, p=0.718)$ PHC (see Fig. 3). Further, there was a non-significant trend for a positive correlation between MWF of the fornix and meanAL $(r=0.325, p=0.079)$. The significant correlation between AL and MWF of the right PHC was followed up by calculating correlations between $\mathrm{AL}$ and $\mathrm{R} 1$ of the right PHC. A non-significant trend was found $(r=0.357$, $p=0.053)$. The correlation between the right $\mathrm{PHC}$ and mean-AL remained significant after controlling for age $(r=0.531, \quad p=0.003)$ and for gender $(r=0.483$, $p=0.008)$. In order to statistically demonstrate the larger magnitude of the correlation between AL and MWF of the right $\mathrm{PHC}$ in comparison to the correlation between $\mathrm{AL}$ 
Fig. 1 Tract "waypoint" regions for the fornix (left) and the parahippocampal cingulum (right) are visualized in green, "NOT" regions are visualized in red

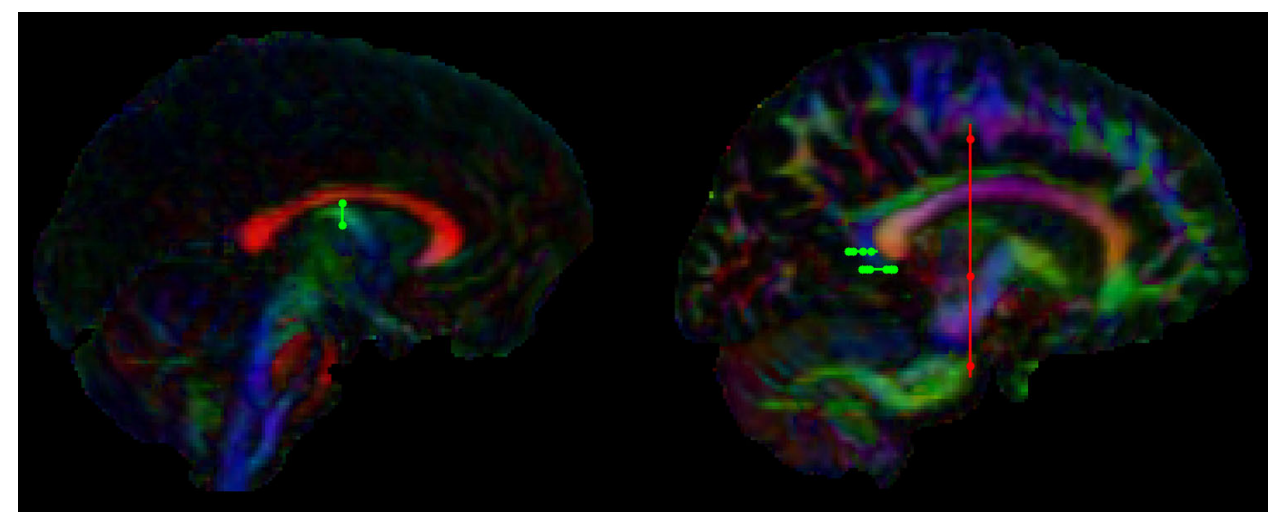

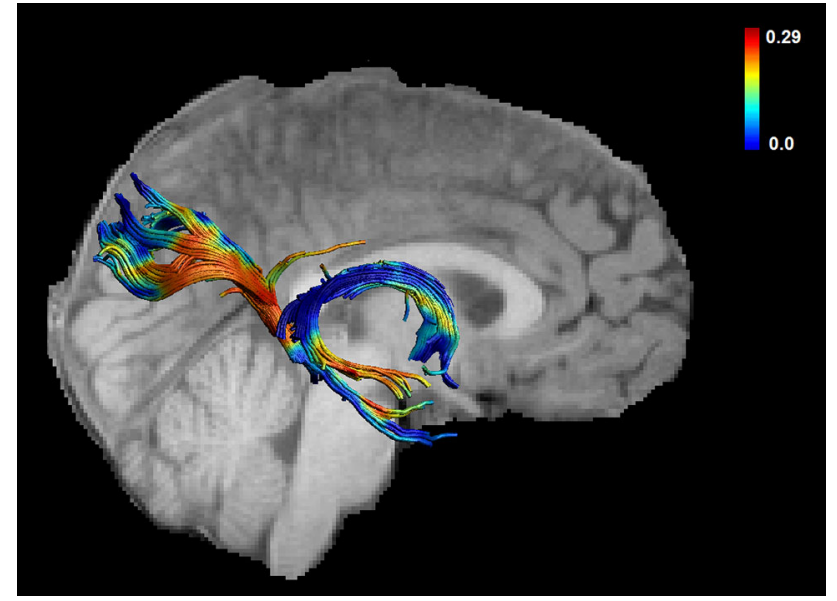

Fig. 2 Myelin water fraction sampled across the fornix and the PHC for one representative subject

and FA of the right PHC Fisher's r-to-z transformation was used $(z=3,6, p=0.0003)$ (Steiger 1980) (http://quantpsy. org/corrtest/corrtest2.htm).

\section{Discussion}

This is the first tractography study linking white matter microstructure of hippocampal pathways to PA. This is also the first study investigating the association between PA and MWF, a more specific measure of myelination than FA. While correlations between FA and AL did not yield significant results for any of the tracts, there was a significant correlation between AL and MWF for the right PHC. Thus, our results suggest that higher PA is associated with higher myelination in the right PHC. We infer, therefore, that MWF not only represents a more specific marker for myelination but is also a more sensitive marker than FA for detecting associations between white matter microstructure and PA. Our results contribute to the understanding of brain-behaviour associations and inter-individual variance even in a highly homogeneous group of young adults.

\section{Myelination of hippocampal pathways and physical activity}

In our study we found a selective positive correlation between PA and MWF in the right PHC. Further, there was a non-significant trend $(r=0.325, p=0.079)$ for a positive correlation between PA and the fornix. Given that the MWF is a putative marker of myelin our findings suggest that higher PA is reflected by higher myelination of the right PHC. Based on the cross-sectional study design it remains unclear if increases in myelination of the right PHC are cause or consequence of increased PA. However, histological studies in animals clearly suggest that experience can influence the degree of myelination. For instance, stress during pregnancy in rodents, the degree of social interactions and the number of play objects all lead to increases myelination (Bennett et al. 1964; Szeligo and Leblond 1977; Markham and Greenough 2004; Wiggins and Gottesfeld 1986). Moreover, electrical stimulation of the premotor cortex in mice has been shown to cause increases in myelination which were associated with improved motor function of the corresponding limb (Gibson et al. 2014). In vitro studies also suggest that neuronal activity by means of neurotransmitter release promotes myelin induction (Demerens et al. 1996; Wake et al. 2011). Further, there is converging evidence from longitudinal neuroimaging studies in humans that PA increases grey matter volume of the hippocampus (Erickson et al. 2011; Pajonk et al. 2010) which may impact plasticity of white matter microstructure as well. Thus, while our study design does not allow for statements regarding causalities of the observed association between MWF and AL, multiple lines of evidence suggest that experience and behaviour indeed induce remodelling of myelination of the brain (Fields 2008). 

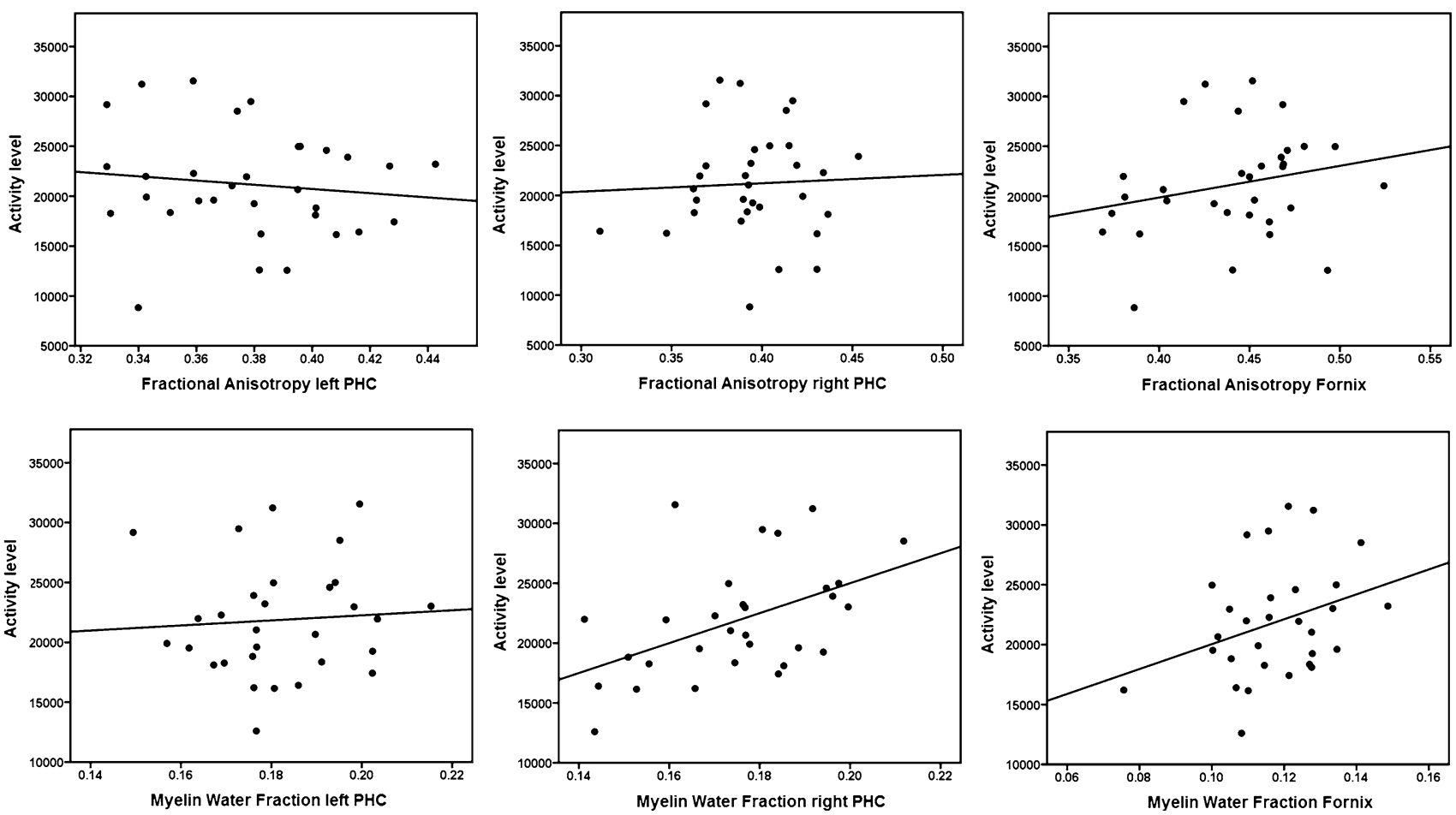

Fig. 3 Correlations between activity levels and fractional anisotropy and between activity levels and myelin water fraction are shown for the left and the right parahippocampal cingulum (PHC) and the fornix

\section{Comparison with previous DTI studies}

We did not find any associations between PA and FA in bilateral PHC and in the fornix. Thus in our sample putative changes in myelin could not be detected using FA. While DT-MRI metrics can be sensitive to differences in myelin (Song et al. 2002) it is worth noting that in genetically modified mice in which myelin has very little presence (e.g. the Shiverer mouse), FA is only lowered around $15 \%$ compared to a wild type mouse (Song et al. 2002). Consequently, changes in myelin of a few percent would have a very small impact on FA. Conversely, as measures such as MWF are thought to be more directly associated with myelin, their sensitivity to myelin changes should be more marked. This may explain why we found a significant correlation between PA and MWF but not between PA and FA.

The absence of a correlation between FA und PA is in line with a voxel-based DT-MRI study of (Walther et al. 2012) who found no association between AL and FA in healthy controls in the PHC. However, there was a negative association of FA and AL in MDD in the left PHC (Walther et al. 2012). A whole brain tract-based spatial statistics (TBSS) (Smith et al. 2006) study found reduced mean diffusivity (MD) in physically fit older adults compared to a less fit control group in a region incorporating the PHC localized in the left hemisphere as well (Tseng et al. 2013).
However, PA was not associated with FA. On the other hand (Burzynska et al. 2014) reported a decrease of FA in sedentary old adults averaged across bilateral PHC using TBSS.

Thus, findings are inconsistent regarding identified diffusion properties (e.g. FA and MD), lateralization and directionality of associations between PA and diffusion properties. In part, this may be owed to differences in study populations. It is possible that in different populations (e.g. students, physically fit or sedentary populations, clinical populations) associations between brain structure and function are reflected by changes in different sub-compartments of white matter microstructure (e.g. changes in axonal diameter, density or myelination) that may in aggregate have different effects on different DT-MRIbased indices (such as FA and MD). However, due to the lack of specificity of DT-MRI-based metrics for white matter sub-compartments this cannot be disentangled. Therefore, the myelin specific measure of MWF represents a significant step forward for the interpretability of white matter neuroimaging studies. A further explanation for those discrepancies is that age impacts on white matter microstructure (Lebel et al. 2010) and neuroplasticity of the PHC (Bracht et al. 2015b). Thus, it is possible that in our young sample changes predominantly occur in myelination which only has a subtle effect on FA (Song et al. 2002), while findings in diffusion properties in elderly 
populations may be the result of different neuroplastic processes (e.g. neuroplasticity of the axons) (Tseng et al. 2013; Burzynska et al. 2014). Moreover, contrasting results between studies may be owed to differences in methodological approaches. For instance, tractography approaches may be more sensitive than voxel-based analyses or regions of interest (ROI)-analyses to detect tract-specific group differences (Bracht et al. 2014, 2015a; Keedwell et al. 2012; Kanaan et al. 2006).

\section{Lateralization}

Our specific finding of an association between PA and MWF of the PHC for the right hemisphere in young students complements findings of associations between PA and diffusion properties (FA and MD) for the left hemisphere in MDD and in an elderly population (Tseng et al. 2013; Walther et al. 2012). Since in contrast to MWF DTMRI-based diffusion properties are not specific for myelin and completely different populations have been investigated using different methodological approaches comparability of the latter findings (Tseng et al. 2013; Walther et al. 2012) with our study in young and healthy participants is limited. It is possible that if those groups had used tractography and looked at MWF as well, a right-sided association between PA and MWF of the PHC would have been identified. Clearly, studies investigating separate homogeneous subgroups with comparable methodological approaches are called for to reliably address the question of differences in lateralization in terms of associations between white matter microstructure and PA.

\section{Clinical implications}

Assuming that PA may indeed induce neuroplastic processes in hippocampal pathways our finding is also of clinical importance. Reduced PA is a clinical feature in various neuropsychiatric disorders with putative hippocampal changes including depression and schizophrenia (Walther 2015; Schrijvers et al. 2008; Bracht et al. 2015c). There is some preliminary evidence that increasing PA (e.g. by means of exercise programs) may help alleviate clinical symptoms (Cooney et al. 2013; Wolff et al. 2011). One may speculate whether clinical improvements following exercise interventions in those disorders are caused by inducing neuroplastic structural changes in pathologically altered projection pathways of the hippocampus (Pajonk et al. 2010). For instance, as suggested by our finding increases in PA may induce myelination of the right PHC. Longitudinal clinical studies using tractography in conjunction with McDESPOT that investigate patient groups at baseline and following an exercise intervention program are required to address this question in future studies.

\section{Summary and conclusions}

In this study we combined an advanced tract-specific approach (dRL) with a myelin specific measure that enables us to draw anatomically meaningful conclusions from our findings (Dell'acqua et al. 2010; Deoni et al. 2008a). A further strength of the study is the homogeneous sample which reduces much of the variance that otherwise is difficult to control for. Our findings suggest that higher PA is associated with higher myelination of the right PHC. Replication studies are called for to validate this initial finding. Given the non-significant trend for an association between PA and MWF of the fornix it would also be of interest to readdress this hypothesis in future studies.

Our results substantially extend previous findings on associations between PA and white matter microstructure. Most importantly, we provide a more myelin-specific measure that allows for a more neurobiologically meaningful interpretation of our data. Furthermore, we chose a tractography-based approach that (in contrast to automated voxel-based approaches) takes individual differences of the course of anatomical pathways into account, and combines multiple samples into the estimate, increasing the statistical sensitivity (Bells et al. 2011). Since the applied dRL-tractography algorithm estimates multiple fibre orientations within a single voxel, inaccuracies that lead to spurious reconstructions if applying DTI-based tractography can be diminished (Dell'acqua et al. 2010).

Since we investigated a homogeneous group of young students our findings may not generalize to a population of non-university students. Further, our study is limited by the cross-sectional study design, which does not allow us to determine whether PA influences white matter microstructure or whether white matter microstructure impacts on motor behaviour. Longitudinal studies are required to address this research question (Scholz et al. 2009). Furthermore, advanced white matter mapping techniques with specificity for axonal properties (e.g. density, diameter) may complement our myelin specific findings (Assaf and Basser 2005).

Acknowledgments This study was supported by the National Centre for Mental Health (NCMH) at Cardiff University, with funds from the National Institute for Social Care and Health Research (NISCHR), Welsh Government, Wales (Grant No. BR09) and the B. Braun Stiftung, Germany, by a Wellcome Trust Investigator Award (to DKJ) and the Swiss National Science Foundation (SNF) (T.B., grant number PBBEP3_144797. We thank Dr. Lisa Brindley and Sonya Foley for discussion about data analyses. 
Open Access This article is distributed under the terms of the Creative Commons Attribution 4.0 International License (http://crea tivecommons.org/licenses/by/4.0/), which permits unrestricted use, distribution, and reproduction in any medium, provided you give appropriate credit to the original author(s) and the source, provide a link to the Creative Commons license, and indicate if changes were made.

\section{References}

Assaf Y, Basser PJ (2005) Composite hindered and restricted model of diffusion (CHARMED) MR imaging of the human brain. Neuroimage 27(1):48-58. doi:10.1016/j.neuroimage.2005.03.042

Bartlett JD, Close GL, MacLaren DP, Gregson W, Drust B, Morton JP (2011) High-intensity interval running is perceived to be more enjoyable than moderate-intensity continuous exercise: implications for exercise adherence. J Sports Sci 29(6):547-553. doi:10. 1080/02640414.2010.545427

Basser PJ, Pierpaoli C (1996) Microstructural and physiological features of tissues elucidated by quantitative-diffusion-tensor MRI. J Magn Reson B 111(3):209-219

Basser PJ, Mattiello J, LeBihan D (1994) Estimation of the effective self-diffusion tensor from the NMR spin echo. J Magn Reson B 103(3):247-254

Basser PJ, Pajevic S, Pierpaoli C, Duda J, Aldroubi A (2000) In vivo fiber tractography using DT-MRI data. Magn Reson Med 44(4):625-632

Beaulieu C (2002) The basis of anisotropic water diffusion in the nervous system: a technical review. NMR Biomed 15(7-8):435-455. doi:10.1002/nbm.782

Bells S, Cercignani M, Deoni S, Assaf Y, Pasternak O, Evans CJ, Leemans A, Jones DK (2011) Tractometry-comprehensive multi-modal quantitative assessment of white matter along specific tracts. In: Proceedings of the International Society for Magnetic Resonance in Medicine Montreal, Canada, p 678

Bengtsson SL, Nagy Z, Skare S, Forsman L, Forssberg H, Ullen F (2005) Extensive piano practicing has regionally specific effects on white matter development. Nat Neurosci 8(9):1148-1150. doi:10.1038/nn1516

Bennett EL, Diamond MC, Krech D, Rosenzweig MR (1964) Chemical and anatomical plasticity brain. Science 146(3644):610-619

Berchtold NC, Chinn G, Chou M, Kesslak JP, Cotman CW (2005) Exercise primes a molecular memory for brain-derived neurotrophic factor protein induction in the rat hippocampus. Neuroscience 133(3):853-861. doi:10.1016/j.neuroscience. 2005.03.026

Bracht T, Heidemeyer K, Koschorke P, Horn H, Razavi N, Wopfner A, Strik W, Walther S (2012) Comparison of objectively measured motor behavior with ratings of the motor behavior domain of the Bern Psychopathology Scale (BPS) in schizophrenia. Psychiatry Res 198(2):224-229. doi:10.1016/j.psychres. 2011.12.038

Bracht T, Horn H, Strik W, Federspiel A, Schnell S, Hofle O, Stegmayer K, Wiest R, Dierks T, Muller TJ, Walther S (2014) White matter microstructure alterations of the medial forebrain bundle in melancholic depression. J Affect Disord 155:186-193. doi:10.1016/j.jad.2013.10.048

Bracht T, Doidge AN, Keedwell PA, Jones DK (2015a) Hedonic tone is associated with left supero-lateral medial forebrain bundle microstructure. Psychol Med 45(4):865-874. doi:10.1017/ S0033291714001949

Bracht T, Jones DK, Muller TJ, Wiest R, Walther S (2015b) Limbic white matter microstructure plasticity reflects recovery from depression. J Affect Disord 170:143-149. doi:10.1016/j.jad. 2014.08.031

Bracht T, Linden D, Keedwell P (2015c) A review of white matter microstructure alterations of pathways of the reward circuit in depression. J Affect Disord 187:45-53. doi:10.1016/j.jad.2015. 06.041

Burzynska AZ, Chaddock-Heyman L, Voss MW, Wong CN, Gothe NP, Olson EA, Knecht A, Lewis A, Monti JM, Cooke GE, Wojcicki TR, Fanning J, Chung HD, Awick E, McAuley E, Kramer AF (2014) Physical activity and cardiorespiratory fitness are beneficial for white matter in low-fit older adults. PLoS ONE 9(9):e107413. doi:10.1371/journal.pone.0107413

Campbell S, MacQueen G (2004) The role of the hippocampus in the pathophysiology of major depression. J Psychiatry Neurosci 29(6):417-426

Cooney GM, Dwan K, Greig CA, Lawlor DA, Rimer J, Waugh FR, McMurdo M, Mead GE (2013) Exercise for depression. Cochrane Database Systematic Rev 9:CD004366. doi:10.1002/ 14651858.Cd004366.Pub6

De Santis S, Drakesmith M, Bells S, Assaf Y, Jones DK (2014) Why diffusion tensor MRI does well only some of the time: variance and covariance of white matter tissue microstructure attributes in the living human brain. Neuroimage 89:35-44. doi:10.1016/j. neuroimage.2013.12.003

Dean DC 3rd, O'Muircheartaigh J, Dirks H, Waskiewicz N, Walker L, Doernberg E, Piryatinsky I, Deoni SC (2014) Characterizing longitudinal white matter development during early childhood. Brain Struct Funct. doi:10.1007/s00429-014-0763-3

Dell'acqua F, Scifo P, Rizzo G, Catani M, Simmons A, Scotti G, Fazio F (2010) A modified damped Richardson-Lucy algorithm to reduce isotropic background effects in spherical deconvolution. Neuroimage 49(2):1446-1458. doi:10.1016/j.neuroimage. 2009.09.033

Demerens C, Stankoff B, Logak M, Anglade P, Allinquant B, Couraud F, Zalc B, Lubetzki C (1996) Induction of myelination in the central nervous system by electrical activity. Proc Natl Acad Sci USA 93(18):9887-9892

den Heijer T, van der Lijn F, Koudstaal PJ, Hofman A, van der Lugt A, Krestin GP, Niessen WJ, Breteler MMB (2010) A 10-year follow-up of hippocampal volume on magnetic resonance imaging in early dementia and cognitive decline. Brain 133:1163-1172. doi:10.1093/brain/awq048

Deoni SCL (2007) High-resolution T1 mapping of the brain at 3T with driven equilibrium single pulse observation of $\mathrm{T} 1$ with high-speed incorporation of RF field inhomogeneities (DESPOT1-HIFI). J Magn Reson Imaging 26(4):1106-1111

Deoni SCL, Ward HA, Peters TM, Rutt BK (2004) Rapid T2 estimation with phase-cycled variable nutation steady-state free precession. Magn Reson Med 52(2):435-439

Deoni SC, Rutt BK, Peters TM (2006a) Synthetic T1-weighted brain image generation with incorporated coil intensity correction using DESPOT1. Magn Reson Imaging 24(9):1241-1248. doi:10.1016/j.mri.2006.03.015

Deoni SCL, Rutt BK, Peters TM (2006b) Synthetic T1-weighted brain image generation with incorporated coil intensity correction using DESPOT1. Magn Reson Imaging 24(9):1241-1248

Deoni SC, Rutt BK, Arun T, Pierpaoli C, Jones DK (2008a) Gleaning multicomponent $\mathrm{T} 1$ and $\mathrm{T} 2$ information from steady-state imaging data. Magn Reson Med 60(6):1372-1387. doi:10. $1002 / \mathrm{mrm} .21704$

Deoni SCL, Rutt BK, Arun T, Pierpaoli C, Jones DK (2008b) Gleaning multicomponent $\mathrm{T} 1$ and $\mathrm{T} 2$ information from steadystate imaging data. Magn Reson Med 60(6):1372-1387

Deoni SCL, Rutt BK, Jones DK (2008c) Investigating exchange and multicomponent relaxation in fully-balanced steady-state free precession imaging. J Magn Reson Imaging 27(6):1421-1429 
Deoni SCL, Williams SCR, Jezzard P, Suckling J, Murphy DGM, Jones DK (2008d) Standardized structural magnetic resonance imaging in multicentre studies using quantitative $\mathrm{T} 1$ and $\mathrm{T} 2$ imaging at $1.5 \mathrm{~T}$. Neuroimage 40(2):662-671

Deoni SC, Mercure E, Blasi A, Gasston D, Thomson A, Johnson M, Williams SC, Murphy DG (2011) Mapping infant brain myelination with magnetic resonance imaging. $J$ Neurosci 31(2):784-791. doi:10.1523/JNEUROSCI.2106-10.2011

Deoni SC, Matthews L, Kolind SH (2013) One component? Two components? Three? The effect of including a nonexchanging "free" water component in multicomponent driven equilibrium single pulse observation of $\mathrm{T} 1$ and $\mathrm{T} 2$. Magn Reson Med 70(1):147-154. doi:10.1002/mrm.24429

Ekkekakis P, Hall EE, Petruzzello SJ (2005) Variation and homogeneity in affective responses to physical activity of varying intensities: an alternative perspective on dose-response based on evolutionary considerations. J Sports Sci 23(5):477-500. doi:10. 1080/02640410400021492

Erickson KI, Prakash RS, Voss MW, Chaddock L, Hu L, Morris KS, White SM, Wojcicki TR, McAuley E, Kramer AF (2009) Aerobic fitness is associated with hippocampal volume in elderly humans. Hippocampus 19(10):1030-1039. doi:10.1002/hipo. 20547

Erickson KI, Voss MW, Prakash RS, Basak C, Szabo A, Chaddock L, Kim JS, Heo S, Alves H, White SM, Wojcicki TR, Mailey E, Vieira VJ, Martin SA, Pence BD, Woods JA, McAuley E, Kramer AF (2011) Exercise training increases size of hippocampus and improves memory. Proc Natl Acad Sci USA 108(7):3017-3022. doi:10.1073/pnas.1015950108

Fedewa AL, Ahn S (2011) The effects of physical activity and physical fitness on children's achievement and cognitive outcomes: a meta-analysis. Res Q Exerc Sport 82(3):521-535. doi:10.1080/02701367.2011.10599785

Fields RD (2008) White matter in learning, cognition and psychiatric disorders. Trends Neurosci 31(7):361-370. doi:10.1016/j.tins. 2008.04.001

Gibson EM, Purger D, Mount CW, Goldstein AK, Lin GL, Wood LS, Inema I, Miller SE, Bieri G, Zuchero JB, Barres BA, Woo PJ, Vogel H, Monje M (2014) Neuronal activity promotes oligodendrogenesis and adaptive myelination in the mammalian brain. Science 344(6183):1252304. doi:10.1126/science.1252304

Goldberg D, Huxley P (1980) Mental illness in the community: the pathway to psychiatric care. Tavistock Publications, London

Goldman-Rakic PS, Selemon LD, Schwartz ML (1984) Dual pathways connecting the dorsolateral prefrontal cortex with the hippocampal formation and parahippocampal cortex in the rhesus monkey. Neuroscience 12(3):719-743

Hanggi J, Koeneke S, Bezzola L, Jancke L (2010) Structural neuroplasticity in the sensorimotor network of professional female ballet dancers. Hum Brain Mapp 31(8):1196-1206. doi: $10.1002 / \mathrm{hbm} .20928$

Heckers S (2001) Neuroimaging and postmortem studies of the hippocampus in schizophrenia. Biol Psychiatry 49(8):4S

Herting MM, Colby JB, Sowell ER, Nagel BJ (2014) White matter connectivity and aerobic fitness in male adolescents. Dev Cogn Neurosci 7:65-75. doi:10.1016/j.dcn.2013.11.003

Heyn P, Abreu BC, Ottenbacher KJ (2004) The effects of exercise training on elderly persons with cognitive impairment and dementia: a meta-analysis. Arch Phys Med Rehabil 85(10): 1694-1704

Hillman CH, Erickson KI, Kramer AF (2008) Be smart, exercise your heart: exercise effects on brain and cognition. Nat Rev Neurosci 9(1):58-65. doi:10.1038/nrn2298

Hurley S, Mossahebi P, Samsonov A, Alexander A, Deoni S, Fisher R, Duncan I, Field A (2010) Multicomponent relaxometry
(mcDESPOT) in the shaking pup model of dysmyelination. In: Proceeding of th 18th Annual Meeting of the ISMRM 4516

Jenkinson M, Smith S (2001) A global optimisation method for robust affine registration of brain images. Med Image Anal 5(2):143-156

Jenkinson M, Bannister P, Brady M, Smith S (2002) Improved optimization for the robust and accurate linear registration and motion correction of brain images. Neuroimage 17(2):825-841

Jeurissen B, Leemans A, Jones DK, Tournier JD, Sijbers J (2011) Probabilistic fiber tracking using the residual bootstrap with constrained spherical deconvolution. Hum Brain Mapp 32(3):461-479. doi:10.1002/hbm. 21032

Jeurissen B, Leemans A, Tournier JD, Jones DK, Sijbers J (2013) Investigating the prevalence of complex fiber configurations in white matter tissue with diffusion magnetic resonance imaging. Hum Brain Mapp 34(11):2747-2766. doi:10.1002/hbm.22099

Johansen-Berg H (2010) Behavioural relevance of variation in white matter microstructure. Curr Opin Neurol 23(4):351-358. doi:10. 1097/WCO.0b013e32833b7631

Johnson NF, Kim C, Clasey JL, Bailey A, Gold BT (2012) Cardiorespiratory fitness is positively correlated with cerebral white matter integrity in healthy seniors. Neuroimage 59(2):1514-1523. doi:10.1016/j.neuroimage.2011.08.032

Jones DK, Horsfield MA, Simmons A (1999) Optimal strategies for measuring diffusion in anisotropic systems by magnetic resonance imaging. Magn Reson Med 42(3):515-525

Jones DK, Christiansen KF, Chapman RJ, Aggleton JP (2013a) Distinct subdivisions of the cingulum bundle revealed by diffusion MRI fibre tracking: implications for neuropsychological investigations. Neuropsychologia 51(1):67-78. doi:10.1016/ j.neuropsychologia.2012.11.018

Jones DK, Knosche TR, Turner R (2013b) White matter integrity, fiber count, and other fallacies: the do's and don'ts of diffusion MRI. Neuroimage 73:239-254. doi:10.1016/j.neuroimage.2012.06.081

Kanaan RA, Shergill SS, Barker GJ, Catani M, Ng VW, Howard R, McGuire PK, Jones DK (2006) Tract-specific anisotropy measurements in diffusion tensor imaging. Psychiatry Res 146(1):73-82. doi:10.1016/j.pscychresns.2005.11.002

Keedwell PA, Chapman R, Christiansen K, Richardson H, Evans J, Jones DK (2012) Cingulum white matter in young women at risk of depression: the effect of family history and anhedonia. Biol Psychiatry 72(4):296-302. doi:10.1016/j.biopsych.2012.01.022

Kitzler HH, Su J, Zeineh M, Harper-Little C, Leung A, Kremenchutzky M, Deoni SC, Rutt BK (2012) Deficient MWF mapping in multiple sclerosis using $3 \mathrm{D}$ whole-brain multicomponent relaxation MRI. Neuroimage 59(3):2670-2677. doi:10.1016/j.neuroimage.2011.08.052

Klein S, Staring M, Murphy K, Viergever MA, Pluim JP (2010) elastix: a toolbox for intensity-based medical image registration. IEEE Trans Med Imaging 29(1):196-205. doi:10.1109/TMI. 2009.2035616

Kolind S, Matthews L, Johansen-Berg H, Leite MI, Williams SC, Deoni S, Palace J (2012) Myelin water imaging reflects clinical variability in multiple sclerosis. Neuroimage 60(1):263-270. doi:10.1016/j.neuroimage.2011.11.070

Laule C, Leung E, Lis DK, Traboulsee AL, Paty DW, MacKay AL, Moore GR (2006) Myelin water imaging in multiple sclerosis: quantitative correlations with histopathology. Mult Scler 12(6):747-753

Lebel C, Caverhill-Godkewitsch S, Beaulieu C (2010) Age-related regional variations of the corpus callosum identified by diffusion tensor tractography. Neuroimage 52(1):20-31. doi:10.1016/j. neuroimage.2010.03.072

Leemans A, Jones DK (2009) The B-matrix must be rotated when correcting for subject motion in DTI data. Magn Reson Med 61(6):1336-1349. doi: $10.1002 / \mathrm{mrm} .21890$ 
Leemans A, Jeurissen B, Sijbers J, Jones DK (2009) ExploreDTI: a graphical toolbox for processing, analyzing and visualizing diffusion MR data. In: Proceedings of the International Society for Magnetic Resonance in Medicine 17th Annual Meeting; Honolulu, Hawaii, p 3536

MacKay A, Whittall K, Adler J, Li D, Paty D, Graeb D (1994) In vivo visualization of myelin water in brain by magnetic resonance. Magn Reson Med 31(6):673-677

Malchow B, Keller K, Hasan A, Dorfler S, Schneider-Axmann T, Hillmer-Vogel U, Honer WG, Schulze TG, Niklas A, Wobrock T, Schmitt A, Falkai P (2015) Effects of endurance training combined with cognitive remediation on everyday functioning, symptoms, and cognition in multiepisode schizophrenia patients. Schizophr Bull. doi:10.1093/schbul/sbv020

Markham JA, Greenough WT (2004) Experience-driven brain plasticity: beyond the synapse. Neuron Glia Biol 1(4):351-363. doi:10.1017/s1740925x05000219

Marks BL, Madden DJ, Bucur B, Provenzale JM, White LE, Cabeza R, Huettel SA (2007) Role of aerobic fitness and aging on cerebral white matter integrity. Ann N Y Acad Sci 1097:171-174. doi:10.1196/annals.1379.022

Marks BL, Katz LM, Styner M, Smith JK (2011) Aerobic fitness and obesity: relationship to cerebral white matter integrity in the brain of active and sedentary older adults. Br J Sports Med 45(15):1208-1215. doi:10.1136/bjsm.2009.068114

Mead GE, Morley W, Campbell P, Greig CA, McMurdo M, Lawlor DA (2009) Exercise for depression. Cochrane Database Syst Rev 3:4366. doi:10.1002/14651858.CD004366.pub4

Metzler-Baddeley C, O'Sullivan MJ, Bells S, Pasternak O, Jones DK (2012) How and how not to correct for CSF-contamination in diffusion MRI. Neuroimage 59(2):1394-1403. doi:10.1016/j. neuroimage.2011.08.043

Metzler-Baddeley C, Baddeley RJ, Jones DK, Aggleton JP, O'Sullivan MJ (2013) Individual differences in fornix microstructure and body mass index. PLoS ONE 8(3):e59849. doi:10.1371/ journal.pone.0059849

Middelkoop HA, van Dam EM, Smilde-van den Doel DA, Van Dijk G (1997) 45-hour continuous quintuple-site actimetry: relations between trunk and limb movements and effects of circadian sleep-wake rhythmicity. Psychophysiology 34(2):199-203

Moore GR, Leung E, MacKay AL, Vavasour IM, Whittall KP, Cover KS, Li DK, Hashimoto SA, Oger J, Sprinkle TJ, Paty DW (2000) A pathology-MRI study of the short-T2 component in formalinfixed multiple sclerosis brain. Neurology 55(10):1506-1510

Mufson EJ, Pandya DN (1984) Some observations on the course and composition of the cingulum bundle in the rhesus monkey. J Comp Neurol 225(1):31-43. doi:10.1002/cne.902250105

Neeper SA, Gomez-Pinilla F, Choi J, Cotman C (1995) Exercise and brain neurotrophins. Nature 373(6510):109. doi:10.1038/ $373109 \mathrm{a} 0$

Nieuwenhuys R, Voogd J, van Huijzen C (2007) The human central nervous system. Springer Fourth Edition

Oldfield RC (1971) The assessment and analysis of handedness: the Edinburgh inventory. Neuropsychologia 9(1):97-113. doi:10. 1016/0028-3932(71)90067-4

Pajonk FG, Wobrock T, Gruber O, Scherk H, Berner D, Kaizl I, Kierer A, Muller S, Oest M, Meyer T, Backens M, SchneiderAxmann T, Thornton AE, Honer WG, Falkai P (2010) Hippocampal plasticity in response to exercise in schizophrenia. Arch Gen Psychiatry 67(2):133-143. doi:10.1001/archgenpsy chiatry.2009.193

Pasternak O, Sochen N, Gur Y, Intrator N, Assaf Y (2009) Free water elimination and mapping from diffusion MRI. Magn Reson Med 62(3):717-730. doi:10.1002/mrm.22055

Razavi N, Horn H, Koschorke P, Hugli S, Hofle O, Muller T, Strik W, Walther S (2011) Measuring motor activity in major depression: the association between the Hamilton Depression Rating Scale and actigraphy. Psychiatry Res 190(2-3):212-216. doi:10.1016/ j.psychres.2011.05.028

Rosenbaum S, Tiedemann A, Sherrington C, Curtis J, Ward PB (2014) Physical activity interventions for people with mental illness: a systematic review and meta-analysis. J Clin Psychiatry 75(9):964-974. doi:10.4088/JCP.13r08765

Scholz J, Klein MC, Behrens TE, Johansen-Berg H (2009) Training induces changes in white-matter architecture. Nat Neurosci 12(11):1370-1371. doi:10.1038/nn.2412

Schrijvers D, Hulstijn W, Sabbe BGC (2008) Psychomotor symptoms in depression: a diagnostic, pathophysiological and therapeutic tool. J Affect Disord 109(1-2):1-20. doi:10.1016/j.jad.2007.10. 019

Sibley BA, Etnier JL (2003) The relationship between physical activity and cognition in children: a meta-analysis. Pediatric Exercise Science 15(3):243-256

Smith SM, Jenkinson M, Johansen-Berg H, Rueckert D, Nichols TE, Mackay CE, Watkins KE, Ciccarelli O, Cader MZ, Matthews PM, Behrens TE (2006) Tract-based spatial statistics: voxelwise analysis of multi-subject diffusion data. Neuroimage 31(4):1487-1505. doi:10.1016/j.neuroimage.2006.02.024

Song SK, Sun SW, Ramsbottom MJ, Chang C, Russell J, Cross AH (2002) Dysmyelination revealed through MRI as increased radial (but unchanged axial) diffusion of water. Neuroimage 17(3):1429-1436

Soundy A, Roskell C, Stubbs B, Probst M, Vancampfort D (2015) Investigating the benefits of sport participation for individuals with schizophrenia: a systematic review. Psychiatr Danub 27(1):2-13

Steiger JH (1980) Tests for comparing elements of a correlation matrix. Psychol Bull 87:245-251

Szeligo F, Leblond CP (1977) Response of the three main types of glial cells of cortex and corpus callosum in rats handled during suckling or exposed to enriched, control and impoverished environments following weaning. J Comp Neurol 172(2):247-263. doi:10.1002/cne.901720205

Tseng BY, Gundapuneedi T, Khan MA, Diaz-Arrastia R, Levine BD, $\mathrm{Lu} \mathrm{H}$, Huang $\mathrm{H}$, Zhang $\mathrm{R}$ (2013) White matter integrity in physically fit older adults. Neuroimage 82:510-516. doi:10. 1016/j.neuroimage.2013.06.011

van der Niet AG, Smith J, Scherder EJ, Oosterlaan J, Hartman E, Visscher C (2014) Associations between daily physical activity and executive functioning in primary school-aged children. J Sci Med Sport. doi:10.1016/j.jsams.2014.09.006

van Praag H, Christie BR, Sejnowski TJ, Gage FH (1999a) Running enhances neurogenesis, learning, and long-term potentiation in mice. Proc Natl Acad Sci USA 96(23):13427-13431

van Praag H, Kempermann G, Gage FH (1999b) Running increases cell proliferation and neurogenesis in the adult mouse dentate gyrus. Nat Neurosci 2(3):266-270. doi:10.1038/6368

van Praag H, Shubert T, Zhao C, Gage FH (2005) Exercise enhances learning and hippocampal neurogenesis in aged mice. J Neurosci 25(38):8680-8685. doi:10.1523/JNEUROSCI.1731-05.2005

Voss MW, Heo S, Prakash RS, Erickson KI, Alves H, Chaddock L, Szabo AN, Mailey EL, Wojcicki TR, White SM, Gothe N, McAuley E, Sutton BP, Kramer AF (2013) The influence of aerobic fitness on cerebral white matter integrity and cognitive function in older adults: results of a one-year exercise intervention. Hum Brain Mapp 34(11):2972-2985. doi:10.1002/hbm.22119

Wake H, Lee PR, Fields RD (2011) Control of local protein synthesis and initial events in myelination by action potentials. Science 333(6049):1647-1651. doi:10.1126/science. 1206998

Walther S (2015) Psychomotor symptoms of schizophrenia map on the cerebral motor circuit. Psychiatry Res Neuroimaging 233(3):293-298. doi:10.1016/j.pscychresns.2015.06.010 
Walther S, Horn H, Razavi N, Koschorke P, Muller TJ, Strik W (2009) Quantitative motor activity differentiates schizophrenia subtypes. Neuropsychobiology 60(2):80-86. doi:10.1159/ 000236448

Walther S, Federspiel A, Horn H, Wiest R, Dierks T, Strik W, Muller TJ (2010) White matter integrity associated with volitional motor activity. NeuroReport 21(5):381-385. doi:10.1097/WNR. 0b013e328337ca29

Walther S, Hugli S, Hofle O, Federspiel A, Horn H, Bracht T, Wiest R, Strik W, Muller TJ (2012) Frontal white matter integrity is related to psychomotor retardation in major depression. Neurobiol Dis 47(1):13-19. doi:10.1016/j.nbd.2012.03.019

Wiggins RC, Gottesfeld Z (1986) Restraint stress during late pregnancy in rats elicits early hypermyelination in the offspring. Metab Brain Dis 1(3):197-203. doi:10.1007/Bf01001781

Wolff E, Gaudlitz K, von Lindenberger BL, Plag J, Heinz A, Strohle A (2011) Exercise and physical activity in mental disorders. Eur
Arch Psychiatry Clin Neurosci 261:186-191. doi:10.1007/ s00406-011-0254-y

Wong AW, Giuffrida L, Wood R, Peckham H, Gonsalvez D, Murray SS, Hughes RA, Xiao J (2014) TDP6, a brain-derived neurotrophic factor-based trkB peptide mimetic, promotes oligodendrocyte myelination. Mol Cell Neurosci 63C:132-140. doi:10.1016/j.men.2014.10.002

Wu M, Chang LC, Walker L, Lemaitre H, Barnett AS, Marenco S, Pierpaoli C (2008) Comparison of EPI distortion correction methods in diffusion tensor MRI using a novel framework. Med Image Comput Comput Assist Interv 11(Pt 2):321-329

Xiao J, Wong AW, Willingham MM, van den Buuse M, Kilpatrick TJ, Murray SS (2010) Brain-derived neurotrophic factor promotes central nervous system myelination via a direct effect upon oligodendrocytes. Neurosignals 18(3):186-202. doi:10. $1159 / 000323170$ 